\title{
Correction to: "Existence, uniqueness and comparison results for BSDEs with Lévy jumps in an extended monotonic generator setting"
}

\section{Christel Geiss · Alexander Steinicke}

Received: 4 July 2019 / Accepted: 4 July 2019

(C) The Author(s). 2019 Open Access This article is distributed under the terms of the Creative Commons Attribution 4.0 International License (http://creativecommons.org/licenses/by/4.0/), which permits unrestricted use, distribution, and reproduction in any medium, provided you give appropriate credit to the original author(s) and the source, provide a link to the Creative Commons license, and indicate if changes were made.

The proof of (Geiss and Steinicke (2018), Theorem 3.5) needs an extra step addressing the problem that our conditions on the generator are not sufficient to guarantee the existence of the considered optional projection:

In Definition 3.3 we defined $f_{n}$ as the optional projection of

$$
(\omega, t, y, z, u) \mapsto{ }^{o,} \mathbb{J}_{f}(n, \omega, t, y, z, u)
$$

with respect to $\mathbb{F}^{n}$ (given by $\mathcal{F}_{t}^{n}:=\mathcal{F}_{t} \cap \mathcal{J}^{n}$ ), with parameters $(y, z, u)$. However, this optional projection does not always exist for generators $f$ satisfying (A1)-(A3).

Sufficient for the existence of the optional projection of a process is boundedness or non-negativity. To guarantee the existence one can replace first $f(\omega, s, y, z, u)$ by

$$
f^{K}(\omega, s, y, z, u)=(-K) \vee f(\omega, s, y, z, u) \wedge K
$$

for some $K>0$.

Clearly, (A1) and (A2) are satisfied for $f^{K}$. Concerning (A3), one observes that only the cases where both factors of $\left(y-y^{\prime}\right)\left(f(s, y, z, u)-f\left(s, y^{\prime}, z^{\prime}, u^{\prime}\right)\right)$ are either positive or negative are relevant. Since

$$
\begin{aligned}
\min \left\{f(s, y, z, u)-f\left(s, y^{\prime}, z^{\prime}, u^{\prime}\right), 0\right\} & \leq f^{K}(s, y, z, u)-f^{K}\left(s, y^{\prime}, z^{\prime}, u^{\prime}\right) \\
& \leq \max \left\{f(s, y, z, u)-f\left(s, y^{\prime}, z^{\prime}, u^{\prime}\right), 0\right\},
\end{aligned}
$$

(A3) is satisfied for $f^{K}$. The above inequality implies that also $(A \gamma)$ holds for $f^{K}$. Hence in order to prove Theorem 3.5, one first starts with $f^{K}$ and $f^{\prime K}$ and gets

$$
Y_{t}^{K} \leq Y_{t}^{\prime K} \quad \mathbb{P} \text {-a.s. }
$$

C. Geiss

University of Jyvaskyla, Department of Mathematics and Statistics, P.O.Box 35, FI-40014 University of Jyvaskyla, Jyvaskyla, Finland

\section{A. Steinicke $(\square)$}

Department of Applied Mathematics and Information Technology, Montanuniversitaet Leoben, Peter Tunner-Straße 25/I, A-8700 Leoben, Austria 
Next we will see that $\left\|Y_{t}-Y_{t}^{K}\right\|$ and $\left\|Y_{t}^{\prime}-Y_{t}^{\prime K}\right\|$ converge to zero for $K \rightarrow \infty$, so that $Y_{t} \leq Y_{t}^{\prime} \quad \mathbb{P}$-a.s. follows. In the proof of Proposition 4.2 it was shown that for data $(\xi, f)$ and $\left(\xi, f^{K}\right)$ it holds

$$
\begin{aligned}
& \sup _{t \in[0, T]}\left\|Y_{t}-Y_{t}^{K}\right\|^{2} \\
& \leq h\left(a, b, 2 \mathbb{E} \int_{0}^{T}\left|Y_{t}-Y_{t}^{K}\right|\left|f\left(t, Y_{t}, Z_{t}, U_{t}\right)-f^{K}\left(t, Y_{t}, Z_{t}, U_{t}\right)\right| d t\right) .
\end{aligned}
$$

To see that the r.h.s. goes to zero, one can use that

$$
\begin{aligned}
& \mathbb{E} \int_{0}^{T}\left|Y_{t}-Y_{t}^{K}\right|\left|f\left(t, Y_{t}, Z_{t}, U_{t}\right)-f^{K}\left(t, Y_{t}, Z_{t}, U_{t}\right)\right| d t \\
\leq & \sqrt{T} \sup _{t \in[0, T]}\left\|Y_{t}-Y_{t}^{K}\right\|\left(\mathbb{E} \int_{0}^{T}\left|f\left(t, Y_{t}, Z_{t}, U_{t}\right)-f^{K}\left(t, Y_{t}, Z_{t}, U_{t}\right)\right|^{2} d t\right)^{1 / 2} .
\end{aligned}
$$

The factor $\sup _{t \in[0, T]}\left\|Y_{t}-Y_{t}^{K}\right\|$ is bounded according to Proposition 4.1, and the integral goes to zero by monotone convergence. Since $\lim _{x \rightarrow 0} h(a, b, x)=0$, one derives that $\lim _{K \rightarrow \infty}\left\|Y_{t}-Y_{t}^{K}\right\|=0$, and in the same way it follows $\lim _{K \rightarrow \infty} \| Y_{t}^{\prime}-$ $Y_{t}^{\prime K} \|=0$.

Moreover, Theorem 3.4 and Lemma 5.1 in Geiss and Steinicke (2018) are only valid, if $f_{n}$ in Definition 3.3 exists. For the proof of Theorem 3.5 this does not cause a problem since we need these results for $f_{n}^{K}$ only.

For more general conditions for the existence of an optional projection than nonnegativity or boundedness we refer to (Dellacherie and Meyer (1982), Remarks VI.44.(f)) and (He et al. 1992).

\section{Authors' contributions}

Both authors read and approved the final manuscript.

\section{Competing interests}

The authors declare that they have no competing interests.

\section{References}

Dellacherie, C., Meyer, P.-A.: Probabilities and potential. B. North-Holland Publishing Co., Amsterdam (1982)

Geiss, C., Steinicke, A.: Existence, uniqueness and comparison results for BSDEs with Lévy jumps in an extended monotonic generator setting. Probab. Uncertain. Quant. Risk. 3(9) (2018)

He, S., Wang, J., Yan, J.: Semimartingale Theory and Stochastic Calculus. Science Press, CRC Press, New York (1992) 